\title{
Barbara Becker:
}

\section{Social robots - emotional agents: \\ Some remarks on naturalizing man-machine interaction}

\begin{abstract}
:
The construction of embodied conversational agents - robots as well as avatars - seem to be a new challenge in the field of both cognitive $\mathrm{Al}$ and human-computer-interface development. On the one hand, one aims at gaining new insights in the development of cognition and communication by constructing intelligent, physical instantiated artefacts. On the other hand people are driven by the idea, that humanlike mechanical dialog-partners will have a positive effect on human-machine-communication. In this contribution I put for discussion whether the visions of scientist in this field are plausible and which problems might arise by the realization of such projects.
\end{abstract}

\section{Agenda:}

Introduction 38

Possible areas of application for "social" robots and "emotional" agents............................................ 38

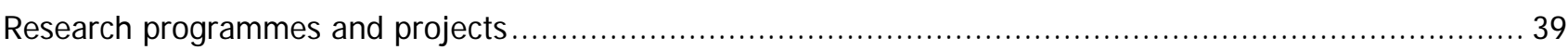

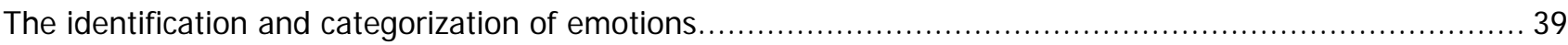

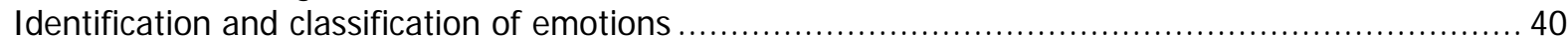

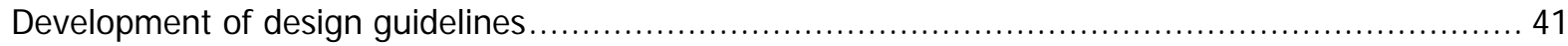

Sense or nonsense of the development of emotional embodied agents ........................................ 41

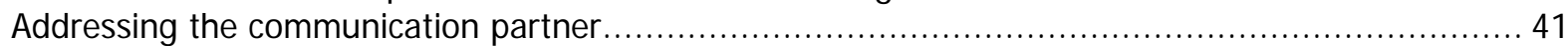

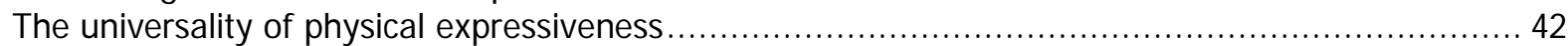

Communication models in visions of future man-machine interactions ............................... 43

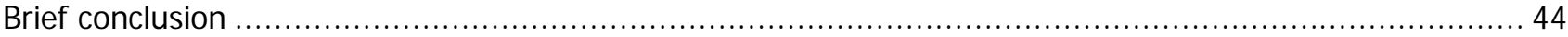

\section{Author:}

Prof. Dr. Barbara Becker:

- Universität Paderborn (UPB) The University for the Information Society, Warburger Str. 100, 33098 Paderborn, Germany

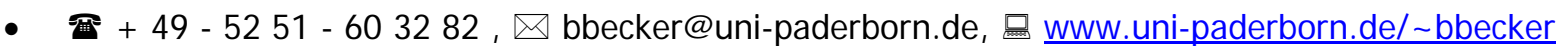


Barbara Becker:

\section{Social robots - emotional agents: Some remarks on naturalizing man-machine interaction}

\section{Introduction}

Traditional Al-concepts, often called "GOFAl (goodold fashioned $\mathrm{Al}$ )“, were dominated by the "physical symbol system hypotheses" according to which cognitive processes might be modelled on a pure symbolic level, ignoring the physical instantiation of the cognitive system. After several years of research, Al-scientists realized that this approach could not solve a basic problem, the socalled symbolgrounding problem. The question how significance emerges in an artefact led to the insight that a cognitive system should be embodied to gain autonomously some experience about the world (Dreyfus 1985, Gold/Engel 1998, Becker 1998, Hayles 1999 and 2003). Accordingly, researchers in this field started to construct little robots which were able to move in limited environments and which were equipped with simple senses (artificial eyes, loudspeakers etc..) (Pfeifer/Scheier 1999, Weber 2003).

While in the beginning, this field was dominated by Al scientists (Brooks 2002, Pfeifer/Scheier 1999, Steels/Brooks 1993), who were mostly interested in the cognitive or technical perspective, some other researchers started to think about possible impacts of this research area on human-computerinteraction (Suchman 1987 and 2004, Wachsmuth/Knoblich 2005, Bath 2003).

In this contribution I would like to concentrate on this last aspect, because current discourses on human-machine interaction increasingly refer to humanoid robots and embodied virtual agents. This impression is reinforced by a number of international and interdisciplinary research projects, some of which are generously financed. The aim of these research projects is to design so-called "believable agents" (Pelauchaud/Poggi 2002) or "sociable robots" (Breazeal 2002), in order to make communication between man and machines "more natural" and to increase people's acceptance of such interactions. Furthermore, attempts are being made to maintain the flow of communication between man and machine for longer periods of times by means of these "embodied emotional agents" (e.g. the European research project "Humaine"), and to intensify the human communication partners' interest in such "dialogues". The perspective "to make interaction between humans and machines more natural" (Wachsmuth/Knoblich 2005) implies some preconditions which are essential in order to be able to develop such a vision in the first place. The ability to address the communication partner in everyday dealings is an important precondition for successful communication processes - in man-machine communications addressing must be possible and it is hoped that it will be made easier by embodied agents. Furthermore, a successful act of communication is always based on the trust the partners have in each other. This aspect should also be taken into account in man-machine interactions. This links in with a further important condition whereby the communication partner is attributed with a form of personality (Cassell 2000), which has a certain degree of stability and continuity over and above the immediate situation.

These important conditions for man-machine interactions were hitherto either not present or insufficiently developed in the interaction between man and machine, and this meant that communication processes, insofar as they took place at all, were quickly terminated. For this reason American (Breazeal 2002, Cassell et al. 2000) and European researchers (Dautenhahn 2004, 2006, Woods 2006, Schröder, Axelsson, Spante and Heldal 2002, 2004), Pelauchaud/Poggi 2000, 2002, Wachsmuth 2005 etc.) began to demand that conversational agents, whether robots or virtual agents, should become more human-like and therefore show emotion in particular, as well as physical forms of interaction (gestures, facial expression, body language) so that they could at least be ascribed a rudimentary form of personality.

In the following I will concentrate on the description and critical analysis of some of these attempts. Particular attention will be paid to the ways in which robots and virtual conversational agents are "emotionalized" and the hopes which are placed in such attempts.

\section{Possible areas of application for "social" robots and "emotional" agents}

How can such a research perspective be justified? A number of areas of application for "social robots" and "emotional agents" are envisaged: Thus such conversational agents could be used in education, 
e.g. in virtual learning situations where ECAs ("embodied conversational agents") communicate the use of educational software to pupils in a userfriendly way. This is also true of supports for dealing with children who have psychological or physical learning and communication difficulties, as can be seen in the AURORE project where autistic children can learn basic communication processes through contact with a simple robot (Woods/Dautenhahn 2006).

A further area of application is in advisory services where it is planned to make information systems more accessible to users when embodied conversational agents assist them in dealing with specific systems (see Wachsmuth et al. 2005). The concept of "social caretaking" which is used in this context (Breazeal 2002), elements of which are planned to be carried out by artifical agents, is a further potential application: The aim is to create robot systems which can help people living alone and in need of assistance insofar as they can carry out simple household tasks and have a monitoring function in order to call for external assistance when necessary.

The area of entertainment is also an important area of application for such systems, as can be seen in the example of various computer games based on avatar technology as well as the (hitherto shortterm) success of small robots (e.g. AlBO) which are used as children's toys. How can such visions be justified, however, and how could they be implemented?

\section{Research programmes and projects}

Various international projects are based on the idea of "humanizing" mechanical artefacts. The vision of developing humanoid systems in this context is most frequently associated with the idea of achieving the personalization of these artefacts by means of the embodiment and emotionalization of robots (e.g. Cassell 2000, Woods 2006 etc.). This type of embodiment takes place either in the form of concrete physical instantiation, i.e. the construction of a robot, or through the creation of a virtual agent who can be addressed as a visible body by the human interaction partner.

Embodiment in this context means not only the physical presence or visual representation of an agent, but includes physical forms of communication. Thus a major focus of such research projects is on the mechanical realization of physical forms of communication such as gestures, facial expression, eyes and posture. Thus they focus on what constitutes a second, non-linguistic and often implicit level of meaning in processes of human interaction (see Wachsmuth / Knoblich 2005). Such robots or agents can produce basic deictic gestures, simple changes of the direction in which they are looking in accordance with the user's position and are able to change their posture. Furthermore, the robots and agents demonstrated a primitive form of facial expression in reaction to the spoken or physical actions of the people interacting with them. Accordingly the aim of the researchers in the "Humaine" project is "to register human emotions, to convey them and to understand the emotional relevance of events" (European project "Humaine, Bath 2004). In order to realize such aims, intensive interdisciplinary co-operation between psychologists, physiologists, philosophers, linguists and computer scientists is necessary (see Wachsmuth et al. 2005). With their specific view of things, researchers from these disciplines identify ways in which emotions are expressed in communication processes and the physical forms of expression with which they correlate, so that these can be reproduced in the relevant systems. However, a number of problems are inherent to this process, only some of which can be discussed in the context of this contribution.

\section{The identification and categorization of emotions}

In the framework of the interdisciplinary European research project "Humaine" the key abilities of an emotional embodied conversational agent are defined as follows:

- the ability to co-ordinate different signs such as gestures, facial expression, posture and language;

- articulateness and expressiveness;

- the generation of affectivity and attentiveness in the communication process.

In order to achieve this, a clear selection and definition of emotions and states of mind is aimed for, which are then related to specific physical, mechanically reproduceable states. Three phases can be distinguished:

- the identification and classification of emotions in a specific communication process; 
- the relation of the emotions considered to be relevant to specific facial expressions, gestures and postures;

- the development of design criteria for the construction of embodied emotional agents.

This process will be examined more closely in the following.

\section{Identification and classification of emotions}

Using different methodological procedures, researchers from different disciplines attempt to identify so-called "basic emotions" and relate these to different physical forms of expression. One study in particular is very frequently cited in projects on the development of ECAs (Scherer 1988). Using pyschological experiments, so-called "basic emotions" were identified, which (it is claimed) can be observed on an intercultural level and which supposedly have universal validity. These include: angry, sad, happy, frightened, ashamed, proud, despairing (Scherer 1988). These feelings are related to a corresponding emotional state which in turn has effects on interpersonal relations, attitudes and affective dispositions. Of particular interest in this context is the attempt to assign such emotions to particular postures and physical forms of expression ${ }^{1}$.A further example is Poggi's $(2005,2006)$ attempt to create lexica of emotions, gestures and physical forms of expression. This is done, for example, using an analysis of video recordings where musicians and conductors are observed in performance. This is supposed to provide information on possible correspondences between facial expressions, gestures and emotions. Poggi sees it as helpful that the music played can provide indications of the emotional states associated with it and the corresponding physical forms of expression. The difficulty of such experiments lies in the untenability of the assumption that there are interculturally typical "basic" emotions which correlate unambiguously with specific physical reactions. Musicians in particular have a tremendous variety of expression so that such lexica must be expanded continually (see also Poggi 2006).

A further popular procedure for identifying physically expressed emotions in communication processes is conversation analysis, where the physical forms of

\footnotetext{
${ }^{1}$ This highly problematic claim to universality will be discussed in more detail later.
}

communication are analysed in correspondence to the respective linguistic message, in order to form an idea of the meaning of non-verbal signs (see André et al. 2005). Such analyses are usually based on a wide range of video recordings: consultations, sales talks, educational communications, artists' performances, politicians' speeches, commercials, television talks etc.

The aim of these conversation analyses is primarily to identify communicative gestures to which specific meanings are attributed, as well as specific, recurring emotions and their physical expression. The aim is to identify unambiguous correlations between emotional states, physical forms of expression and semantic messages.

The following discussion is concerned with the component of emotionality, whereby facial expressiveness is of particular interest, the factor on which Poggi (2006) concentrates in her studies. As already mentioned above, she uses video recordings to try to identify unambiguously classifiable emotions and to relate these to specific facial expressions.

The result of this research is a complex pattern of correlations between emotions and physical forms of expression: Thus, for example, anxiety, panic and fear are associated with the following facial expression: open mouth, teeth visible, lips tense, eyes wide open, eyebrows linear (Poggi 2006). In contrast, grief, depression, and sorrow are associated with a different facial expression: corners of mouth turned down, eyebrows angled inwards and eyelids lowered. Joy, contentment and desire are associated with shining eyes, laughing mouth and slightly raised eyebrows, while anger, aggression, disgust and rage are correlated with a screwed up nose, wrinkled forehead, down-turned mouth and wide open eyes (op cit.).

These decontextualized and generalized attributions are recorded in tables in which the emotions considered relevant to communication processes are associated with specific facial expressions ${ }^{2}$. Overall it should be noted that these correlations of mental conditions with specific forms of expression and behaviour are largely directed towards observable and describable phenomena and that therefore

${ }^{2}$ The difficulties associated with such a degree of de-individualization and decontextualization will be discussed in greater detail later. 
such tabular attributions mean that the complexity of subjective feelings and the variety of associated physiological processes are only taken into account in a highly reductionist manner ${ }^{3}$.

\section{Development of design guidelines}

Tables such as those developed by Poggi are the basis both for the conception of virtual agents and the development of robotic faces. These systems show certain reactions of facial expression in interactive situations as they are equipped with programmes which can analyse the semantic content of a message at a very basic level. If, for example, one "speaks" to the ECA "MAX" developed at the University of Bielefeld, it reacts not only verbally but also by means of facial expression. MAX responds to insults verbally and with a "sad" facial expression which corresponds to the classifications described above. ECAs such as GRETA (Pelachaud et al. 2002), ROBOTA (Dautenhahn et al. 2006), KISMET (Breazeal 2002) etc. react in a similar manner. They suggest, especially to the inexpert user, a form of emotionality on the part of the artificial agent which is intended to motivate the human user not to end the "communication" process too soon. Longer-term empirical studies are necessary to show how these effects should be evaluated and whether the constructors' hopes will be fulfilled in the long term.

For the time being the expressiveness of such agents can be summarised as follows: The "emotionality" of virtual agents is expressed in extremely reduced "facial expresssions" which are limited to observable behaviour and obviously do not correspond to an emotional level of experience. Robots and virtual agents neither experience the feelings that their expressions transport in a reduced form, nor do their feel the physiological reactions that frequently correspond to such emotions (racing heart, rise in blood pressure, breathlessness, relaxation). This is particularly apparent in their expressionless mechanical voices and empty eyes, both symbols of a non-existent personality ${ }^{4}$. This obvious deficit justifies questioning the point of such "emotionalization" of agents. If, as is the case with most researchers in this field, one does not have extravagant expectations and does not assume

${ }^{3}$ If such aspects are referred to at all, which is not usually the case.

4 The relevance of voice and eyes as a sign of personality will be discussed later. that artificial systems can have a form of emotionality comparable with that among humans, then the emotional reaction of the agents remains a mere surface effect which is easily seen through. Therefore the following discussion is not concerned with whether robots or virtual agents will at some stage actually have emotions or whether their embodiment corresponds to the complex human body or ever will do so. Instead, the question is how social practice changes, i.e. how people deal with such agents that suggest emotionality and embodiment. The first empirical findings are already available (Axelsson 2002, Ball/Breese 2000, Dautenhahn/Woods 2006), although these must be continually added to as the findings will continue to change in step with very rapid technological development. Therefore the following observations remain provisional.

\section{Sense or nonsense of the development of emotional embodied agents}

Let us return to the initial motivation of the researchers in this field: Firstly, there is the attempt to make communication between man and artefact "more natural"; secondly there is the hope that the personalization and emotionalization of these artificial agents will enable the flow of communication to be maintained for longer, as these agents can be seen as "trustworthy" interaction partners (Churchill 2000), (Pelachaud/Poggi 2002), (Woods 2006).

In the following I would like to discuss a few aspects which in my opinion should be viewed critically: the problem of addressability; the modelling and universalization of emotionality; the concept of communication between man and machine.

\section{Addressing the communication partner}

An important and plausible reason for such experiments lies in the potential addressability ${ }^{5}$ of the respective partner, which is especially relevant in communication situations where the interaction partners can communicate via avatars. It is possible to use avatars to find out quite quickly whether a

${ }^{5}$ Addressability in the sense that a supposedly concrete partner exists to whom specific characteristics can be ascribed. 
certain person is in a virtual space and ready to communicate there. Likewise, an advisory or tutorial agent can be perceived as a visible and therefore addressable authority which can possibly better cushion possible frustration when dealing with information and training systems than an unidentifiable partner.

Studies of communication processes in chats comparing purely text-based chats and those using avatar technology showed that the presence of persons in the shared communication fora could be established faster by using avatars, so that interrupted communication processes could easily be taken up again (Becker/Mark 1999). The avatars made it easier to address the other person, to localize him in the shared virtual space and to determine his social position within the entire communication scenario (see also Schroeder 2002, Axelsson 2002, Spante 2004, Heldal, 2004). In a different way, studies carried out in the context of the "Aurora" project (see Dautenhahn et al 2004, 2006) showed that even the simplest robot systems, equipped with minimal gestures and facial expressions, could encourage autistic children to limited reactions, imitation and interaction. These children seemed, insofar as this could be identified, to develop a form of relationship to these robot systems with their simple facial expressions and gestures, a relationship which was noticeably less fearful than their relationships with people. They "interacted" with these systems which were addressable via their physical presence, by imitating the robots' simple movements and following the robots' "gaze". However, it remains questionable whether this legitimizes using such systems for therapeutic purposes, as is being considered in the context of the project.

The personalization of robots and avatars, which is in common particularly observed among children and is obviously reinforced by even primitive expressions of feelings (see Cassell 2000, Breazeal 2002, and also Dautenhahn 2004), is highly ambivalent. On the one hand the personalization of avatars can frequently lead to more rapid initiation of communication and reinforce the feeling of social involvement (Schröder et al. 2002). On the other hand, however, "emotionalized" robots or avatars suggest the existence of an emotional context on the part of the virtual agent or robot, which is a pure fiction.

This brings us back to the issue of social practice (Gamm 2005): How do people, e.g. children, deal with the artificial agents that give them the impres- sion of having feelings similar to those they have themselves? The personalization frequently observed when dealing with agents is unproblematic as long as the users can maintain a reflective distance from such attributions. Nor does this present a serious conflict for children, as long as the artificial agents have the status of cuddly toys or dolls, to which a form of personality has always been ascribed. Nevertheless, children usually had a clear perception of the artificiality of these toys. The future will show whether this changes when the artefacts show expressive reactions or can move ${ }^{6}$. However, once agents with the superficially reproduced form of emotionality described above are used seriously for therapeutic ends or even as "social caretakers", the problem of such attributions becomes more acute. Even the choice of the term "social robots" feeds an illusion that can reinforce the idea of a social practice, according to which artificial agents are perceived as equal interaction partners (Blow 2006). Corresponding visions are to be found, for example, where there are speculations about new forms of democracy between man and artefacts or where the agent metaphor is applied in an undifferentiated way to people and artefacts (Suchman 2004). The social and psychological effects of these new forms of communication, and projective attributions on the part of the human users can, however, only be described in speculative terms at the moment.

\section{The universality of physical expressiveness}

The second criticism refers to the modelling of physical forms of expression, especially of emotional behaviour. It has already been mentioned above that the research now taking place in this context is mostly limited to the observable behavioural level. Emotions are linked to specific physical reactions, while the feelings behind these and subjective experience are scarcely taken into account. If one examines psychological and physiological research in the wide field of emotions in this context, three roughly differentiated research orientations can be distinguished: theories which concentrate on the cognitive mental level and examine the sphere of subjective experience and the conscious perception of certain emotions; theories which are limited to the observation of the physiological manifestations of emotions (heartbeat, breathing, skin reactions etc.); theories which are interested in the behav-

${ }^{6}$ Long-term research projects are necessary here. 
ioural level and study facial expression, posture, gestures and actions.

It is apparent that behaviourist research, concentrating on behavioural patterns, is the fundamental basis for the conception of emotionally embodied agents and robots. However, this involves a not unproblematic scientific re-orientation. This affects the entire field of Cognitive Science, whose advantage lays in its very rejection of behaviourism. Furthermore it corresponds with new trends towards naturalization, which are especially evident in the supremacy of genetic engineering and neurosciences.

Apart from these problems, a further difficulty can be observed: If one looks again at the processes used to identify and classify emotions, the weakness of every type of scientific modelling becomes apparent: Observable emotions and apparently associated behavioural patterns are subject to selective perception implicit in the observation process: One sees what one wants to see. This implies that the observed correlations between a certain emotion and specific, associated behaviours also depend on the viewpoint of the observer and his interests. Attempts such as Poggi's (2006) to create lexica for correlations between emotional states, certain observable behavioural patterns and physical manifestations are therefore always confronted with the problem of having to critically examine their own observer's perspective.

Attempts to identify timeless, interculturally relevant "basic emotions", and to associate these with behavioural patterns which are equally universally valid, also come up against limiting factors when the complexity and individuality of emotional expressiveness, both on the experienced level and in terms of behaviour, can scarcely be expressed in abstract models. The decontextualization and abstraction intrinsic to modelling become a problem when such models are used for the conception of virtual agents or robots which reproduce uniform and stereotyped patterns of emotionality. Normative processes such as are to be observed everywhere, are aggravated by such projects. However, it is to be expected that the polysemy of emotional expression will produce a similar variety of attributions of meaning on the part of human intepreters ${ }^{7}$. The wide range of interpreta-

${ }^{7}$ This can be seen in the observation of photographic portraits and their interpretation by different tions once again indicates the limits to the modelling of emotionality, and especially its artifical reproduction.

In spite of the hope that human communication partners are capable of individual interpretations and a critical distance when dealing with these artefacts $^{8}$, a certain unease remains. If one calls to mind the significance of a concrete partner, especially in the socialization of children, then the simplified forms of expression and the "empty" eyes of artficial agents are more problematic. Processes corresponding to Lacan's Mirror Stage (Lacan 1973) whereby the $I$ is constituted in the regard of others, are apparently evoked here, but do not really take place. It is less the absence of such "responses" by other people which is criticized here, but rather the suggestion that the artificial agent is a genuine opposite / partner. In this context the attempts at personalization seem to open up an area of potential conflicts.

\section{Communication models in visions of future man- machine interactions}

The concept of communication underlying such attempts need to be examined. It seems as though most concepts of man-machine interactions still start with the assumption of a simple transmitterreceiver model, according to which the message sent by the transmitter arrives at the receiver exactly as originally intended and is interpreted in accordance with the transmitter's intentions. In contrast to this are reciprocal concepts of communication $^{9}$, according to which the speaker's intention is already coloured by the implicit invitation of the addressee. Furthermore, in these new approaches it is assumed that the interpretation of the "message" on the part of its recipients always takes place in the context of their specific experiences, i.e. it varies individually and is context-dependent to a marked degree and only corresponds in a limited way to the intentions of the "transmitter". ${ }^{10}$

viewers as well as in the analysis of attributions of emotions in communication processes.

${ }^{8}$ Such hopes are repeatedly expressed in the context of Cultural Studies, see Hall 1980.

9 E.g. as developed by Levinas, Waldenfels and Bauman.

${ }^{10}$ Reference to the approaches of Cultural Studies (e.g. Hall etc.) is helpful in this context. 
This reciprocity, which can be considered typical of man-machine interactions, assumes a form of physicality and personality from which virtual agents and robots are far removed. Voice and eyes as two essential elements of embodied communication are paid far too little attention in the current approaches: Robots' eyes or the "eyes" of a virtual agent are empty; they reveal the absence of any type of personality and show that their minimal emotional expressions do not correspond to an "inner" life ${ }^{11}$. Reduced to purely superficial effects and stereotyped, simplified forms of "expression", such empty faces do not evoke that form of responsiveness which is typical of man-man communication. And this is even true in cases where people do not look at each other.

The same is true of voices: Whenever robots or virtual agents have a voice, this almost always seems lifeless. Their dynamics are in accordance with stereotyped models of the melodics of spoken language, which do not call forth any response from their human partner. A feeling of being addressed can at most be observed at the level of the simple exchange of information; a sense of being addressed by a physical partner, which causes something in me to respond, be it negatively or positively, i.e. which evokes that form of resonance leading to a reciprocal communication relation, does not occur (see Waldenfels 1999).

This would not be a problem if potential social conflicts did not thereby arise. Responsiveness in communication, which always involves an awareness of and response to the needs of one's partner as well as the achievement of one's own intentions, is insofar of significance in that the concept of responsiveness intimates the element of responsibility. By implicitly or explicitly sensing the needs of the other person and reacting to these as a communication partner in whatever form, one accepts responsibility for the communicative situation and for the other person (Bauman 2003). This right of the other person, communicated largely through eyes and voice as well as language and gestures (Levinas 1999), is not present in man-machine interaction and is therefore not repeatedly experienced, which would be the necessary basis for a corresponding

11 The concept "inner life" suggests a pre-discursive stable self. This is naturally not the case, as the inner life referred to here is largely constituted in the act of interaction with a person's environment and other people. sensitization. The possible social consequences can at the moment only be described in speculative terms.

\section{Brief conclusion}

How, then, should projects for the construction of humanoid robots and emotionally embodied agents be evaluated? Although this type of research is only beginning, some cautious can be formulated. The assumption inherent in the concept of the agent and even more so in that of the autonomous robot, according to which these artefacts can be conceived as independent actors and accordingly used, is highly questionable. Instead, I would argue in favour of a relational perspective which does not primarily see virtual agents and robots in the context of their possible similarity to humans and potential personalization. In my opinion it is of greater interest to examine the extent to which such artefacts are localized in various social networks and what specific functions they could assume here in accordance with their abilities (see also Suchman 2004). The anthropomorphization of such agents (Gamm 2005) would be irrelevant in such a context, as their abilities would emerge from the agents' position within the social networks.

In accordance with this viewpoint one could take leave of the perspective which interprets virtual agents, ECAs and robots as human-like interaction partners. Instead, they could take on specific tasks within a relational behavioural concept, which would accord them an empowerment to act which is limited to these tasks. This would do justice to the potential of these supposedly "intelligent" artefacts while allowing for their limitations. In this context, however, it is doubtful whether there is any point to the surface simulation of emotionality in robots and conversational agents or whether it merely encourages fictions which could become problematic.

\section{References}

André, E. et al.: "Employing Al methods to control the behavior of animated interface agents", in: AAl 13: 415-448

Axelsson, A.S.: "The same as being together?", Chalmers, Göteburg 2002

Ball, G./Breese, J.: "Emotion and Personality in a conversational agent", in: Cassell, J. et al. "Embodied conversational agents", MIT press, Cambridge 2000 
Bauman, Z.: "Flüchtige Moderne", Frankfurt 2003

Bath, C.: "Einschreibungen von Geschlecht", in: Weber, J./Bath, C. (eds.) "Turbulente Körper, soziale Maschinen", Opladen 2003

Becker, B.: "Leiblichkeit und Kognition", in: Gold, P./Engel, A.K. Hrsg): "Der Mensch in der Perspektive der Kognitionswissenschaft", Frankfurt 1998

Becker, B./Mark, G.: "Constructing social systems through computer-mediated communication", in: Virtual reality 4, 1999

Blow, M. et al. "The art of designing Robot facesdimension for human-robot interaction", HRI 2006, Utah 2006

Breazeal, C.L., "Designing sociable robots", MIT 2002

Cassell, J. et al. "Embodied conversational agents", MIT press, Cambridge 2000

Churchill, E.F. et al. "May I help you?: Designing Embodied Conversational Agents Allies", in: Cassell, J. et al. "Embodied conversational agents", MIT press, Cambridge 2000

Dautenhahn, K. et al.: "How may I serve you? A robot companion approaching a seated person in a helping context", in HRI '06, UTAH, 2006

Dautenhahn, K./Werry, I.: "Towards interactive robots in autism therapy! ", in: Pragamatics and cognition 12:1, 2004, p. 1-35

Dreyfus, H.: "Die Grenzen künstlicher Intelligenz", Königstein 1985

Gold, P./Engel, A.K. Hrsg): "Der Mensch in der Perspektive der Kognitionswissenschaft", Frankfurt 1998

Gamm, G./Hetzel, A. (Hrsg): "Unbestimmtheitssignaturen der Technik", Bielefeld 2005, hier: Vorwort

Hall, S. "Cultural Studies: Two Paradigms", in: Media, Culture and Society 2, 1980

Hayles, K.: "How we became posthuman", Chicago/London 1999

Hayles, K.: "Computing the human", in: Weber, J./Bath, C. (eds.) "Turbulente Körper, soziale Maschinen", Opladen 2003

Heldal, 1: "The usability of collaborative virtual environments", Göteburg 2004

Lacan, J.: "Schriften I", Olten 1973

Levinas, E.: "Die Spur des Anderen", München 1999

Pelachaud, C./Poggi, l.: "Subtleties of facial expressions in embodied agents", in: The journal of visualization and computer animation, 2002, 13, p. 1-12

Pfeifer, R./Scheier, C.: "Understanding Intelligence", Cambridge Mass. 1999

Poggi, I., Pelachaud, C.: "Performative facial expressions in animated faces", in: Cassell, J. et al. "Embodied conversational agents", MIT press, Cambridge 2000

Poggi, l., "Towards the alphabet and the lexicon of gesture, gaze and touch", in: Konferenzmaterialien zur FG "Embodied communication in humans and machines", ZiF, Bielefeld 2005

Poggi, I. "Le parole del corpo", Rom 2006

Robins, B. et al.: "Robots as embodied beings", University of Hertfordshire, 2005

Scherer, K.R. "Faces of Emotion: Recent Research", Hillsdale, N.J. 1988

Schroeder, R. (ed): "Social life of avatars", Göteburg 2002

Spante, M.: "Shared virtual environments", Göteburg 2004

Suchman, L.: "Plans and situated actions", Cambridge 1987

Suchman, L. "Figuring Personhood in Sciences of the Artificial", Lancaster 2004

Wachsmuth, I. /Knoblich G. "Embodied Communication in Humans and Machines", ZiF-Mitteilungen, Bielefeld 2005

Waldenfels, B.: "Die Vielstimmigkeit der Rede", Frankfurt 1999

Weber, J./Bath, C. (eds.) "Turbulente Körper, soziale Maschinen", Opladen 2003

Weber, J.: "Umkämpfte Bedeutungen", Frankfurt 2003

Woods, S. et al.: "Is this robot like me? Links between Human and Robot personality traits", Hertfordshire 2006 